\title{
Mesenchymal stromal-cell transplants induce oligodendrocyte progenitor migration and remyelination in a chronic demyelination model
}

\author{
J Jaramillo-Merchán ${ }^{1}$, J Jones ${ }^{1}$, JL Ivorra ${ }^{2}$, D Pastor ${ }^{3}$, MC Viso-León ${ }^{1}$, JA Armengól ${ }^{4}$, MD Moltó \\ E Geijo-Barrientos ${ }^{1}$ and S Martínez ${ }^{*, 1}$
}

Demyelinating disorders such as leukodystrophies and multiple sclerosis are neurodegenerative diseases characterized by the progressive loss of myelin that may lead toward a chronic demyelination of the brain's white matter, impairing normal axonal conduction velocity and ultimately causing neurodegeneration. Current treatments modifying the pathological mechanisms are capable of ameliorating the disease; however, frequently, these therapies are not sufficient to repress the progressive demyelination into a chronic condition and permanent loss of function. To this end, we analyzed the effect that bone marrowderived mesenchymal stromal cell (BM-MSC) grafts exert in a chronically demyelinated mouse brain. As a result, oligodendrocyte progenitors were recruited surrounding the graft due to the expression of various trophic signals by the grafted MSCs. Although there was no significant reaction in the non-grafted side, in the grafted regions oligodendrocyte progenitors were detected. These progenitors were derived from the nearby tissue as well as from the neurogenic niches, including the subependymal zone and dentate gyrus. Once near the graft site, the cells matured to myelinating oligodendrocytes. Finally, electrophysiological studies demonstrated that axonal conduction velocity was significantly increased in the grafted side of the fimbria. In conclusion, we demonstrate here that in chronic demyelinated white matter, BM-MSC transplantation activates oligodendrocyte progenitors and induces remyelination in the tissue surrounding the stem cell graft.

Cell Death and Disease (2013) 4, e779; doi:10.1038/cddis.2013.304; published online 29 August 2013

Subject Category: Experimental Medicine

Demyelinating disorders are a group of diseases characterized by myelin loss. The cause for this destruction is varied, such as an autoimmune attack (i.e., multiple sclerosis (MS) $)^{1,2}$ or metabolic/genetic alterations (leukodystrophy). ${ }^{3}$ The loss of myelin ultimately causes neuronal disruption, as the oligodendrocytes are crucial for both the metabolic support of the axons ${ }^{4}$ as well as the correct transmission of the nerve impulse.

Oligodendrocyte progenitor cells (OPCs), which express the NG2 proteoglycan, ${ }^{5}$ can be detected migrating and differentiating into mature oligodendrocytes as early as 7 days after a demyelinating lesion. ${ }^{6}$ These cells are extensively distributed in the central nervous system (CNS), are capable of dividing throughout the lifespan and are activated when there is a demyelinating process. ${ }^{7}$

MS can be divided into two main stages: acute and chronic. Although in the acute phase the nearby OPCs are capable of remyelinating the damaged axons, ${ }^{8-10}$ in the chronic phase the cell's migratory and differentiating capabilities are altered, resulting in sustained and progressive demyelination. ${ }^{11}$ This is at least partly because of the lack of factors that recruit and stimulate remyelinating properties of OPCs. ${ }^{12}$ Thus, the objective of this work was to develop a remyelinating therapy in chronically demyelinated white matter based on activation of existing OPCs to induce migration and differentiation.

To this end, there are a number of studies concerning trophic-based activation of OPCs (reviewed by Rivera et $a l^{13}$ ). Many of the identified trophic factors are known to be produced and secreted by bone marrow-derived mesenchymal stromal cells (BM-MSCs). Previous reports have proven that these cells are capable of inducing regeneration, neuroprotection and functional recovery in an MS mouse model. ${ }^{14-16}$ Though the cause for this effect in vivo is not well understood, there is evidence in vitro that BM-MSCs are capable of stimulating the proliferation and differentiation of neural progenitors toward an oligodendrocyte lineage. ${ }^{17,18}$

\footnotetext{
${ }^{1}$ Neuroscience Institute, University Miguel Hernandez-Spanish Council for Scientific Research (UMH-CSIC), Alicante, Spain; ${ }^{2}$ Department of Genetics, University of Valencia, CIBERSAM, INCLIVA, Valencia, Spain; ${ }^{3}$ Sports Science Research Center, University Miguel Hernandez, Elche, Spain and ${ }^{4}$ Department Physiology, Anatomy and Cell Biology, Pablo de Olavide University, Seville, Spain

*Corresponding author: S Martínez, Neuroscience Institute, University Miguel Hernandez-Spanish Council for Scientific Research (UMH-CSIC), Avda. Ramon y Cajal s/n. 03550, San Juan, Alicante, Spain. Tel: +34 96 5919556; Fax: +34 96 5919555; E-mail: smartinez@umh.es

Keywords: mesenchymal stromal cells; demyelinating mouse model; trophic release; oligodendrocyte activation; remyelination

Abbreviations: BDNF, brain-derived neurotrophic factor; BM-MSC, bone marrow-derived mesenchymal stem cell; CC, corpus callosum; CNS, central nervous system; cSEZ, caudal subependymal zone; DG, dentate gyrus; DMEM, Dulbecco's modified Eagle's medium; FGF, fibroblast growth factor; GFAP, glial fibrillary acidic protein; GFP, green fluorescent protein; IGF, insulin-like growth factor; LV, lateral ventricle; MBP, myelin basic protein; MS, multiple sclerosis; MSC, mesenchymal stem cell; NGF- $\beta$, nerve growth factor $\beta$; NT3, neurotophic factor-3; NT4/5, neurotrophic factor-4/5; OPC, oligodendrocyte progenitor cell; PB, phosphate buffer; PDGF, plateletderived growth factor; PFA, paraformaldehyde; SEZ, subependymal zone; TEM, transmission electron microscopy

Received 08.4.13; revised 08.7.13; accepted 16.7.13; Edited by Y Shi
} 
Here we describe the use of BM-MSC in a model of chronic demyelination. This approach will give us insight as to the mechanisms by which BM-MSCs may activate OPCs in vivo, and ultimately induce remyelination and axonal conduction improvement.

\section{Results}

MSCs activate oligodendrogenesis and remyelination. After 12 weeks of cuprizone-embedded diet, the mice presented clear demyelinating lesions in the corpus callosum (CC) and fimbria (Figures $1 \mathrm{~b}-\mathrm{d}$ ). However, although in the $\mathrm{CC}$ both myelinated and non-myelinated patches were detected (Figure 1c), the fimbria was rather homogeneously non-myelinated (Figure 1d). Thus, the surgical procedures were decided to be performed in this white matter structure.
As early as 2 weeks after stereotaxic-guided intra-fimbrial transplantation (Figure 2a), NG2-positive OPCs as well as mature oligodendrocytes (myelin basic protein $(\mathrm{MBP}+)$ ) were detected surrounding the grafted cells (Figures $2 b-d$ ). The percentage of NG2, MBP and glial fibrillary acidic protein (GFAP) immunoreactivity was calculated and compared in the sham controls and stem cell-treated groups (Figures $2 e-h$ ). Concerning NG2, there was a slight increase in expression in the sham-treated group compared with its contralateral side, although not as remarkable as that observed in the stem celltreated group, where over 10 times more NG2 was detected (Figures $2 \mathrm{e}$ and $\mathrm{f}$ ). This was also observed in both MBP and GFAP staining, where the stem cell-injected fimbria presented higher levels of expression of these myelin and astrocyte markers, respectively, compared with the contralateral side (Figures $2 \mathrm{e}, \mathrm{g}$ and $\mathrm{h}$ ). The sham-treated fimbria did not differ

a

\begin{tabular}{llll|}
\hline DEMYELINATION & ANALYSIS \\
\hline CUPRIZONE 0,2\% TREATMENT & RECOVERY & \\
\hline day: -90 & day: 0 & day: 90 \\
& MSC grafted & \\
SHAM operated & & \\
\hline
\end{tabular}
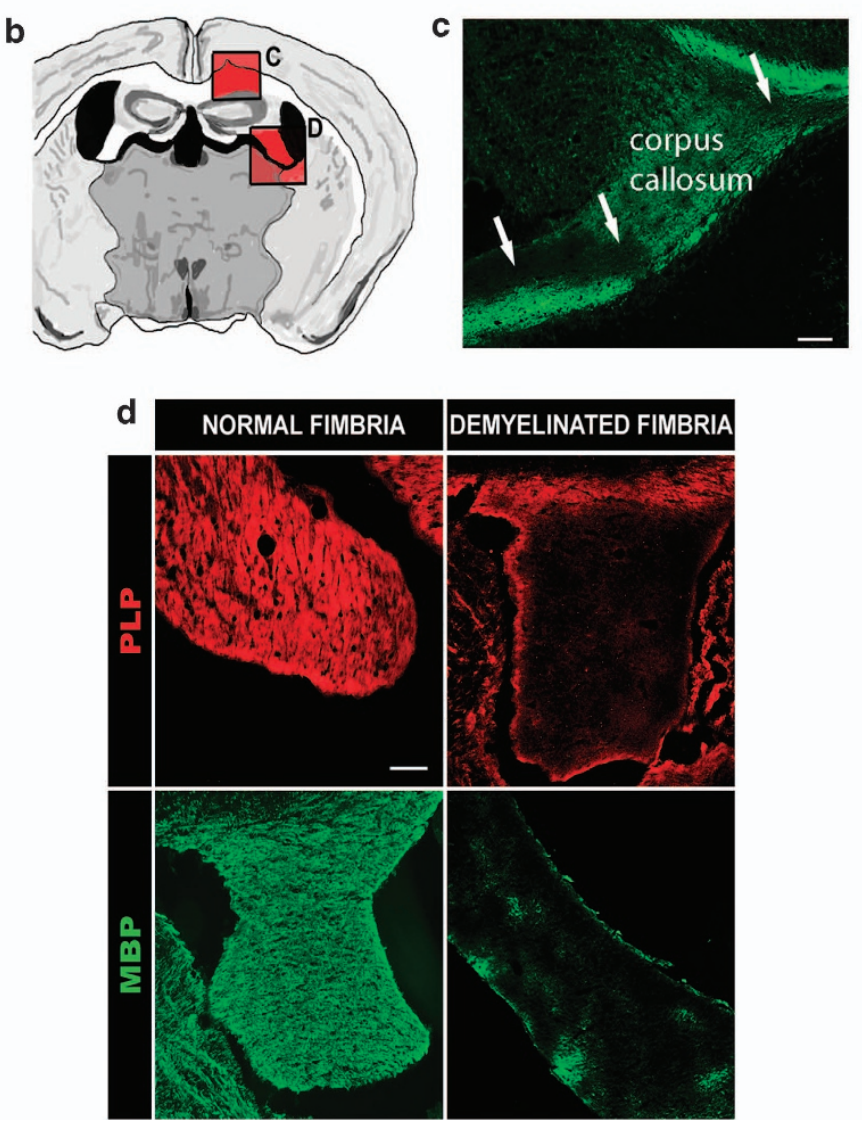

Figure 1 Effect of chronic intake of cuprizone in the CNS. (a) Chronogram of the experimental protocol. (b) Schematic representation of a transveral section of the brain, indicating with red squares the $\mathrm{CC}$ (c) and fimbria (d). (c) Immunohistochemistry of a transveral section of the CC of a mouse treated for 3 months with cuprizone and stained for MBP (in green). Arrows indicate patches of demyelination (MBP-negative), similar to that seen in chronic lesions of MS. (d) Proteolipid protein (PLP) (red) and MBP (green) staining in the fimbria of a non-treated mouse and of a cuprizone-treated mouse of the same age. Note the lack of expression of the two myelin-forming proteins, indicating a homogenous demyelination process of the fimbria. Scale bar, $75 \mu \mathrm{m}$ (c and $\mathbf{d})$ 
with its contralateral side in MBP staining, whereas for GFAP a slight increase was detected.

The results shown here indicate that there was a significant increase in OPCs and mature oligodendrocytes in the fimbria treated with MSCs, which in turn gave rise to newly formed myelin.

MSC transplantation increases the conduction velocity of demyelinated fibers. Axon conduction velocity was measured in order to confirm if the newly formed myelin around the MSC graft was functional (Figures $3 a-d$ ). In the recordings obtained with electrodes placed in the vicinity of the graft (see Materials and Methods), we detected two different populations of axons corresponding to fast- and slow-conducting axons (Figure $3 e$ ); in normal mice the average conduction velocity of these components was close to 1 and $0.4 \mathrm{~m} / \mathrm{s}$, respectively (measured at room temperature). The slow conduction fibers were absent when the region of the fimbria near the hippocampus was registered (Figure 3f).

The values of conduction velocity of fast-conduction fibers are given in Figure $3 \mathrm{~g}$. In normal (wild type), control demyelinated and sham-operated animals, the conduction velocity of the fast and slow (not shown) fibers was similar in all the regions tested (only rostral and caudal registered regions are shown). However, in stem cell-treated mice the conduction velocity of the fast fibers was almost twice as fast in the rostral region compared with the other groups $(t=3.11$; $P$-value $=0.032$ ), although the caudal region had increased values, though not statistically significant. The conduction velocity of the slow fibers did not change in any experimental condition (data not shown).

Thus, our electrophysiological analysis indicated that MSC transplants increased axon conduction speed of the area in the fimbria surrounding the graft. This effect is higher in the anterior region of the fimbria, which represents the axonal segments localized closer to the caudal subependymal zone (cSEZ) in relation to the graft.

MSCs activate oligodendrocyte progenitors by releasing trophic factors. As commented in the Introduction section, several authors have indicated that MSCs are capable of releasing a number of trophic factors that activate OPCs and induce their differentiation toward myelinating oligodendrocytes. Several factors that are known to induce this effect in OPCs were analyzed in MSCs both in vitro (before transplantation, Figure 4a) and in vivo (Figure 4b). As a result, neurotrophic factor-4/5 (NT4/5), neurotophic factor-3 (NT3), platelet-derived growth factor (PDGF) and nerve growth factor- $\beta$ (NGF- $\beta$ ) were detected both in vitro and in vivo. The contralateral (control) fimbria did not show any significant immunoreactivity of any of the factors analyzed. There was no immunoreactivity detected for these trophic factors in the sham group (data not shown). The combinations of several of these growth factors have been previously shown to be capable of inducing remyelination in cuprizone-treated mice. ${ }^{19}$

As no transdifferentiation or cell fusion processes were observed (data not shown), we conclude that the activation of native OPCs and oligodendrocytes was due to the release of at least these trophic factors by the grafted stem cells.
MSCs stimulate oligodendroglial differentiation and migration of cells from the SEZ and dentate gyrus of the hippocampus. The results shown so far indicate an activation of OPCs in the fimbria due to the paracrine effect of several trophic factors from the grafted MSCs. However, this increase in OPCs derives not only of nearby areas surrounding the fimbria, but possibly from the neurogenic niches, the SEZ and the dentate gyrus (DG) of the hippocampus. This phenomenon is possible because of the fact that the ventricular surface of the $C C$, caudal septum and fimbria coincide in the dorso-caudal pole of the lateral ventricle (LV), which advances over the ventricular surface of the hippocampal formation.

In the case of the SEZ, a number of NG2-positive cells were detected migrating from this region in the side of the fimbria where the stem cells were injected (Figure 5a). These cells presented cytoplasmic prolongations with a rostro-medial to caudo-lateral orientation and followed the SEZ of the right LV, with practically no invasion of the alveus or outer cortical hippocampal structures (Figures $5 b-f$ ).

To further confirm if the NG2-positive cells detected from the SEZ toward the fimbria originated in this area, Nilo1 expression was analyzed (Figure $5 \mathrm{~g}$ ). This marker is specific for neural precursors of the SEZ. ${ }^{20}$ As a result, the vast majority of the NG2-positive cells also expressed Nilo1, confirming that they derive from the SEZ (cells in purple). Migrating NG2 + cells were not detected in the contralateral side of the fimbria, where no stem cells were injected (Figures 5h and i).

Also, the subgranular layer of the DG was analyzed in longitudinal sections, as this neurogenic area is relatively close to the grafted site (Figures $6 \mathrm{a}$ and b). Stronger MBP immunostaining was detected in the rostral fimbria near the DG with respect to more caudal areas of the fimbria (Figures $6 \mathrm{c}$ and d). Also, some cells seemed to detach from the distal pole of DG subgranular and migrated through the subepial region (Figures $6 e-g$ ), to finally invade the nearby fimbria parenchyma and even extend prolongations that surrounded the axons, similar to that observed in mature oligodendrocytes (Figures $6 \mathrm{~h}-\mathrm{j}$ ). These NG2 + cells from the subgranular zone of the DG were Nilo1-negative (data not shown).

\section{Discussion}

In this work, we analyzed the effect that MSC grafts have in a chronic demyelinated model. As a result, OPCs, identified as $\mathrm{NG} 2$ + cells, increased in number and migrated from the nearby neurogenic niches toward the graft, which include cells from the subependymal (NG2+/Nilo + ) and subgranular (NG2 + /Nilo - ) zones (Figure 7). Furthermore, these progenitor cells matured into oligodendrocytes and remyelinated the surrounding area. This seemed to be due to the effect of various trophic factors secreted by the grafted MSCs, inducing OPC migration toward demyelinated white matter and differentiation to ultimately promote remyelination.

Toxicity-induced demyelinating models such as cuprizone are similar to the lesions that appear in MS, with massive apoptosis of oligodendrocytes; however, if the treatment is prematurely suspended, spontaneous remyelination occurs. This physiological remyelination occurs in two phases: 
(A) OPC proliferation and colonization of the demyelinated area and $(B)$ differentiation toward immature oligodendrocytes that contact with demyelinated axons, followed by OPC maturation and remyelination. This process is regulated by a complex mechanism of coordinated expression of signaling factors (reviewed by Chari et al..$^{8,9}$ ).

OPCs are normally generated in the SEZ and DG of the hippocampus in adult animals. During development, OPCs
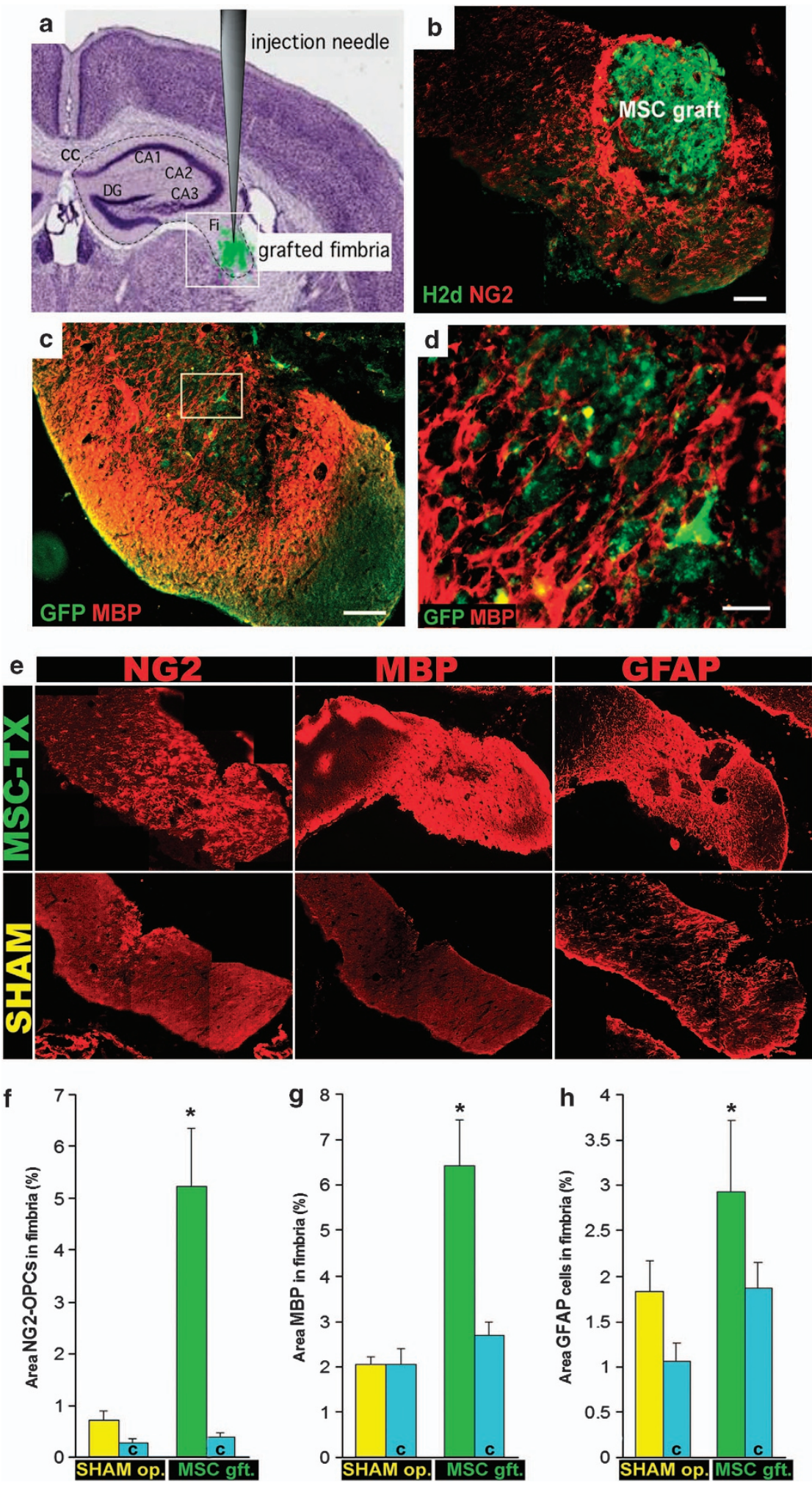
originate and migrate from the embryonic subventricular zone into the forebrain parenchyma. ${ }^{7}$ Caudally, these precursors migrate into the fimbria and hippocampus. Therefore, the fimbria represents an ideal area of interest to explore remyelination because of its localized source of OPCs from the cSEZ.

OPCs are constitutively present in the adult CNS and can specifically differentiate in vivo toward remyelinating oligodendrocytes. However, in some myelin diseases, such as in the primary progressive and evolution of relapsing-remitting MS, there is a multiple dysfunction in the remyelinating mechanisms that characterize the appearance of chronic lesions, ${ }^{21}$ similar to that observed in chronic cuprizone-treated mice. $^{22}$ This may be because of either an absence of nearby OPCs or that they are quiescent and do not react to the signals that normally appear during the early stages of demyelination. In both cases, they may be due to, at least partly, the lack of factors that activate the necessary mechanisms to induce regeneration. Thus, it is of great importance to stimulate the quiescent OPCs, as well as induce their migration to the affected area.

There are numerous growth factors known to induce remyelination, including $P D G F,{ }^{23}$ fibroblast growth factors (FGFs), ${ }^{24} \mathrm{NT3}^{25}$ and insulin-like growth factor (IGF)-1. ${ }^{26,27}$ Also, certain combinations of growth factors work synergistically to repair and remyelinate. For example, FGF2 along with IGF activate OPCs, ${ }^{28}$ whereas PDGF convert adult OPCs, which divide slowly, to 'newborn' progenitors with a higher proliferation rate. ${ }^{29}$

However, there are certain disadvantages in using trophic factors alone as a possible treatment. One of the most important disadvantages is to find the proper dosage. An insufficient amount of the trophic factor would not have any effect on the remyelination process, but an excess may actually cause further damage. This was observed, for example, with brain-derived neurotrophic factor (BDNF) infusion after an axotomy in rats. ${ }^{30}$ In this work, high doses of the trophic factor inhibited motorneuron axonal regeneration. At the clinical level, ALS patients were administered intrathecally different doses of BDNF, causing an overall worsening compared with placebo. ${ }^{31}$ This cited article also shows another possible problem with growth factor treatment: the method of administration. Although oral intake may cause little to no effect on the tissue of interest, especially because many growth factors are not capable of surpassing the bloodbrain barrier, direct injection or using micro-pumps has shown that the necessary concentration of the trophic factor to induce regeneration or neuroprotection may cause side effects that surpass the possible benefits. For example, NGF infusion in Alzheimer's disease patients resulted in a very slight cognitive amelioration but accompanied by constant back pain that only disappeared when the treatment was halted. ${ }^{32}$ In addition, GDNF intraventricular delivery has been used in Parkinson's disease, which resulted in no clinical benefits and unwanted side effects. ${ }^{33}$ The importance of adverse events demonstrate the biological activity of neurotrophic factors in human's neurodegenerative conditions and reveal the importance of adequate delivery pattern (continuous instead of intermittent) close to neuronal targets in physiological concentrations.

MSCs, on the other hand, are known to secrete a large array of factors. ${ }^{34}$ These factors are released in an autocrine and paracrine way in response to the niche they are placed, in order to regenerate or protect nearby degenerating tissue. In MS, MSC transplantation presents an additional benefit, which resides in the modulation and suppression of the immunological response that characterizes this disease by inhibiting and regulating T-cell proliferation, as well as suppresses the inflammatory response. ${ }^{35}$

Some of the factors that MSCs release are known to promote the differentiation of neural progenitor cells toward oligodendrocytes, ${ }^{36}$ establishing an oligodendrocytic niche. We describe the expression of an array of growth factors. These trophic factors released by the MSC reach the neurogenic niches to promote OPC recruitment toward the source releasing them. These OPCs are active as they express NG2 and migrate from the caudal pole of the LV SEZ homolateral to the transplant. This stream of cells continues up to the graft, without abandoning the subependymal niche. Also, some OPCs from the DG of the hippocampus were observed.

Interestingly, in our sham controls we observed a slight increase in OPCs around the injection site. A previous study described that OPCs are activated when there is an ischemia, demyelination or other lesion in the CNS. ${ }^{37}$ However, these OPCs were not capable of differentiating into mature oligodendrocytes nor initiate any reactive myelin formation, demonstrated by the absence of MBP immunoreaction.

Even though we observe the activation and maturation of OPCs in a chronic demyelinated tract, it is necessary to confirm that functional remyelination occurs. In chronic lesions of MS, the oligodendrocytes that survive in a demyelinating lesion and have lost their myelinating expansions are not capable of contributing to the remyelination, despite being capable of forming new myelin layers, because they cannot place these layers in the demyelinated axons (reviewed by Franklin ${ }^{38}$ ). Thus, it is necessary to perform electrophysiological studies in order to confirm that the remyelination process observed here results in a functional recovery. In this work, we measured the conduction velocity of several areas in the periphery of the transplant. Under normal

Figure 2 OPCs around the graft site. (a) Diagram depicting the location of the injection site. The fimbria (Fi) is adjacent to the CC and the hippocampus, subdivided into CA1, CA2 and CA3. (b) MSC-grafted fimbria immunostained for H2d (green) and NG2 (red) in the injection site. In all cases, the graft stimulated OPC clustering. (c) Stem cell-treated fimbria immunostained for GFP (green) and MBP (red) in the injection site. (d) Close-up image of a region of the periphery of the graft (inset in c) staining for MBP (red) and GFP (green) where the stem cells can be seen intertwined with myelinated fibers. (e) NG2, MBP and GFAP expression (in red) in the fimbria of mesenchymal stem cell-grafted mice, sham-operated and the control demyelinated fimbria of the grafted mice. Note that the NG2 in MSC was taken 1 mm rostrally from the graft where no MSC were present. ( $\mathbf{f}-\mathbf{h})$ Quantification of the expression of NG2 (f), MBP $(\mathbf{g})$ and GFAP $(\mathbf{h})$ in the fimbria of MSC-grafted mice (green bars), sham-operated (yellow bars) and the control fimbria (blue bars) of both groups. A significant increase of the NG2 expression $(t=-3.19 ; P$-value $=0.013)$, MBP expression $(t=-4.34 ; P$-value $=0.004)$ and GFAP expression $(t=-2.60 ; P$-value $=0.032)$ was detected in the MSC-grafted mice compared with the sham-operated mice, whereas no significant differences were detected in both control demyelinated fimbria (corresponding to the contralateral side of the fimbria) in sham-operated and control demyelinated fimbria in MSC-grafted. Scale bar, $100 \mu \mathrm{m}$ 

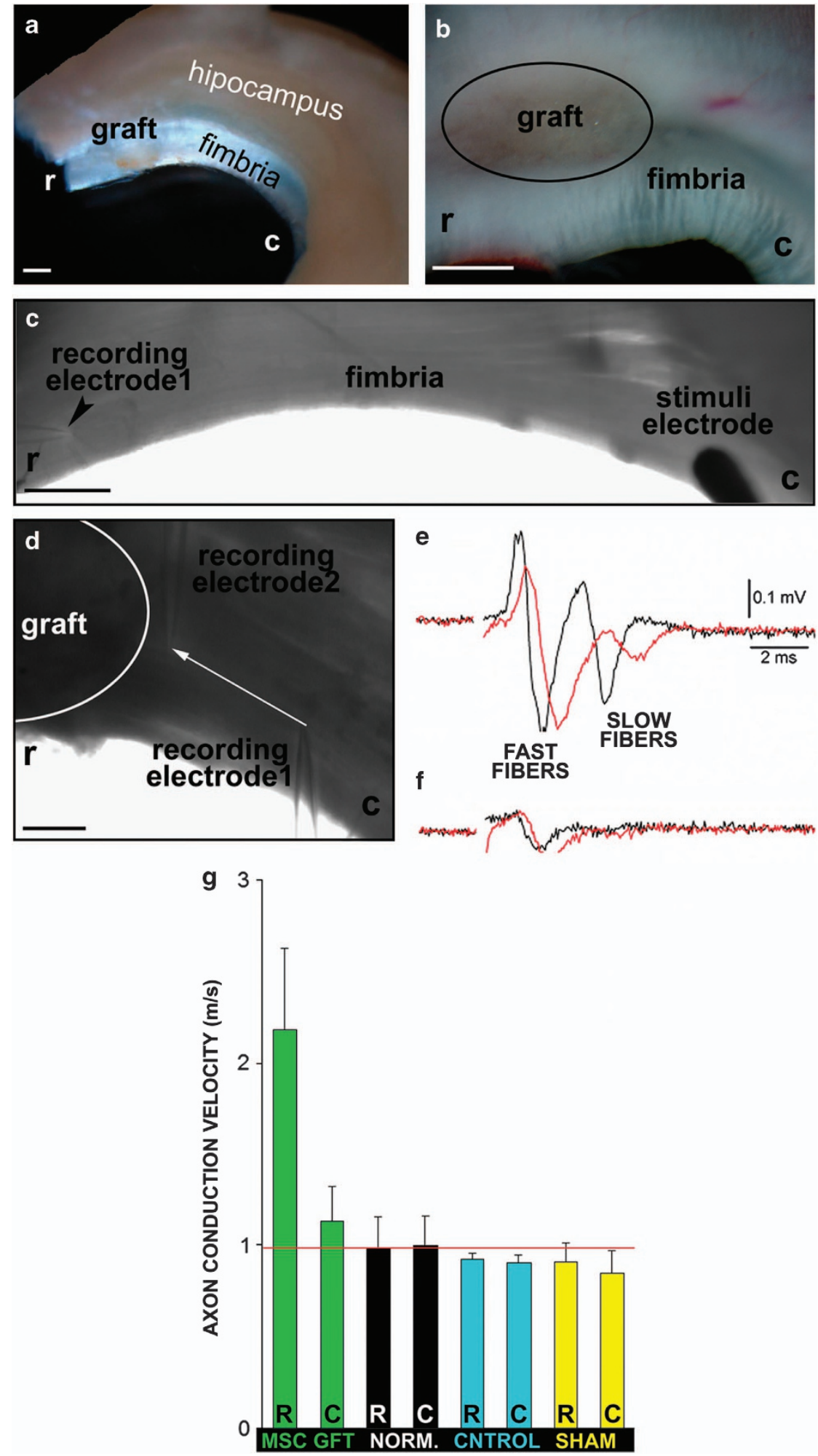

Figure 3 Axon conduction velocity in various regions of the fimbria. (a and $\mathbf{b}$ ) Images of the extracted fimbria, depicting the location of the graft. (c) The stimulus electrode located in the caudal region (c) of the fimbria and a registry electrode in the rostral area (caudal to the graft) ( $r$ ). (d) Details of the position of the two registry electrodes to obtain the recordings of the caudal fibers around the graft (white line). (e and f) Two fibers were detected: fast (thicker myelin sheaths) and slow (thin and/or non-myelin sheaths) fibers. (g) Histogram depicting the axon potential velocities in the fast fibers measured in the MSC (green), normal non-demyelinated (black), sham-operated (yellow) and control demyelinated fimbria (blue) in the rostral (R) or caudal (C) regions. Red line denotes wild-type values. Scale bar, $1 \mathrm{~mm}(\mathbf{a}$ and b); $500 \mu \mathrm{m} \mathrm{(c);} \mathrm{and} 100 \mu \mathrm{m}(\mathbf{d})$ 


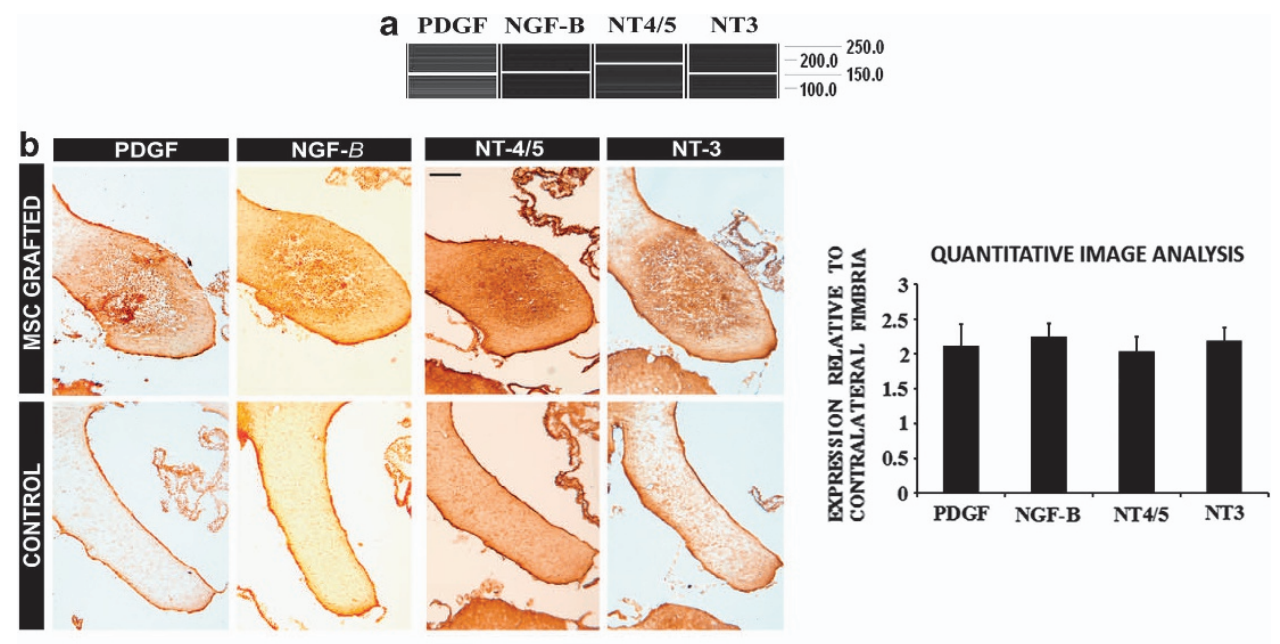

Figure 4 Trophic factor expression after grafting. (a) PCR analysis of cultured mesenchymals stromal cells. (b) Immunohistochemical analysis of the stem cell-treated fimbria for the expression of various trophic factors compared with the control fimbria. NT4/5, NT3, PDGF and NGF- $\beta$ were expressed in MSC-grafted fimbria. The histogram to the right depicts the quantification of the immunoreactivity of the trophic factors analyzed by immunohistochemistry, relative to the contralateral (non-treated) side of the fimbria. A value over 1 indicates a higher immunoreactivity in the treated side of the fimbria compared with the non-treated side. Scale bar, $50 \mu \mathrm{m}$

circumstances, two fiber types can be detected: fast and slow fibers, corresponding to axons with thick and thin myelin sheaths, respectively. Interestingly, in our model after demyelination the conduction velocities of both fast and slow fibers were not severely affected compared with normal values. This was possibly due to the spontaneous compensatory mechanisms in the axon, such as axonal dilatation that increases axonal diameter. In the MSC-treated group, the conduction velocity of the fast fibers doubled with respect to the other groups, including wild-type controls. Also, when the rostro-medial area of the fimbria was registered, close to the fornix, we observed a very significant increase in the conduction velocity, possibly due to the proximity of OPC migratory routes deriving from the neurogenic niches. In this area a large concentration of OPCs was observed. This strong increase of impulse transmission could be due to the remyelination of dilated axons, which could restore saltatory conduction in already dilated axons.

In conclusion, MSC transplantation resulted in the activation of nearby oligodendrocyte progenitors, which because of the cuprizone treatment maintained an unactivated state. In turn, these OPCs matured into myelinating oligodendrocytes. This was due to the secretion of several trophic factors, which also activated the neurogenic niches, provoking the migration of OPCs from the subventricular zone and DG of the hippocampus toward the graft. These results indicate a possible stem cell-based therapy where mesenchymal stem cells may be delivered into the CNS of MS in the chronic phase of the lesions.

\section{Materials and Methods}

Animals. All experiments have been performed in compliance with the Spanish and European Union laws on animal care in experimentation (Council Directive $86 / 609 / E E C)$ and have been analyzed and approved by the Animal Experimentation Committee of our University. Green fluorescent protein (GFP) (C57BI/6- Tg(ACTB-EGFP)1Osb/J) transgenic mice were used as donors, whereas $\mathrm{C} 3 \mathrm{H} / \mathrm{He}\left(\mathrm{H} 2 \mathrm{~K}^{\mathrm{k}}\right)$ mice were used as hosts. Both animal strains were bred and maintained in our animal facilities.
Demyelinating treatment. Four-week old $\mathrm{C} 3 \mathrm{H} / \mathrm{He}$ mice were given a diet with $0.2 \%$ cuprizone $(\mathrm{w} / \mathrm{w})$ for 12 weeks ad libitum in order to obtain a chronic version of toxicity-induced demyelination, as previously indicated, ${ }^{39}$ but with the following changes. Finely powdered cuprizone (Sigma-Aldrich, St. Louis, MO, USA) was dissolved in $60 \%$ tap water (w/v) with $0.5 \%$ (w/v) commercialized cane sugar. The cane sugar was added to avoid the weight loss observed in other studies because of the cuprizone treatment. After 12 weeks, the mice were grafted with MSCs (or culture medium in the case of sham-operated group) and returned to normal diet until their death, which was depending on the test at 15 or 90 days (see timeline in Figure 1a).

Isolation and culture of MSCs. The isolation and culture of MSCs was performed as in our previous studies. ${ }^{40,41}$ Briefly, the bone marrows of transgenic GFP mice were extracted, disaggregated and cultured in plastic flasks. The following culture medium was used: high-glucose Dulbecco's modified Eagle's medium (DMEM) (Invitrogen, Life Technologies, Paisley, UK), 15\% fetal bovine serum (Biochrom AG, Berlin, Germany) and 1\% penicillin/streptomycin (SigmaAldrich). The stem cells were maintained for 3-4 weeks (passages 2-3) before proceeding to the surgical interventions.

Cell transplantation and experimental groups. A total of 24 mice were injected with MSCs $(n=10$ for immunohistochemistry, $n=8$ for electrophysiology and $n=6$ for electron microscopy). Also, for the sham-operated group, another 10 animals were used $(n=4$ for immunohistochemistry, $n=3$ for electrophysiology and $n=3$ for electron microscopy). In both groups, before performing the surgical procedure, $0.1 \mathrm{mg} / \mathrm{kg}$ of buprenorphine (Buprex, ScheringPlough, Madrid, Spain) was injected into the mice. Isoflurane (Esteve Veterinary, Milan, Italy), an inhalational anesthesia, was used, and the mice was placed on a stereotaxic apparatus (Stoelting, Wheat Lane Wood Dale, IN, USA). The animal was monitored, and anesthesia concentration was controlled. The injections were performed in the right fimbria at the following stereotaxic coordinates (Kopf, model 900 small animal stereotaxic instrument; anteroposterior relative to bregma, mediolateral and dorsoventral from the surface of the brain): $-0.11,-0.16$ and $-0.26 \mathrm{~mm}$, respectively. The MSCs were injected using a $5 \mu \mathrm{l}$ Hamilton syringe (Reno, NV, USA) coupled with a DMEM-rinsed siliconed-capillary glass. In the MSC-operated group, 300000 cells were transplanted in $2 \mu \mathrm{l}$ of DMEM. The left fimbria was considered as the internal control of demyelination for all experimental groups and in all studies except in electron microscopy, where the side of the graft was randomized. After the injection, the incision was sutured and the mice were monitored throughout the whole process of experimentation. For the immunohistochemical analysis, the mice were killed at 15 days after the surgical intervention. As for the remaining tests (quantitative PCR, electrophysiology and electron microscopy), the mice were killed 3 months after the intervention. 

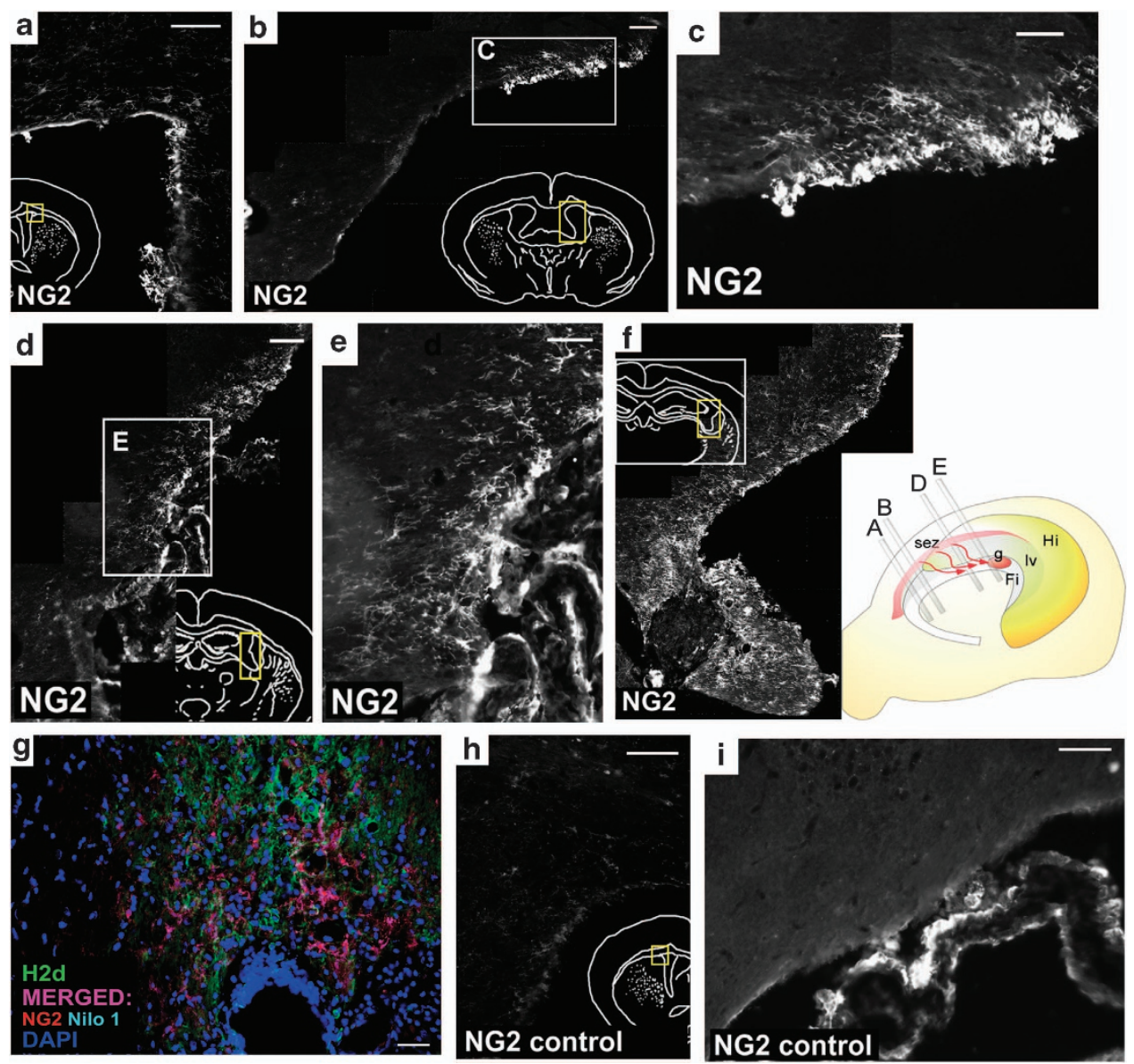

Figure 5 Analysis of the neurogenic niches: subventricular zone. (a-f) Coronary sections of the LV showing NG2 + cells migrating from the subventricular zone to the MSC-grafted fimbria. NG2 + cells, corresponding to OPCs, migrate rostro-caudally from the subventricular zone in the side of the fimbria where the stem cells were injected (a). The cells migrated bordering the lateral ventricular until they reached the fimbria (b-f). (b) Immunohistochemical image depicting the NG2 + cells bordering the LV. (c) Close-up of inset in b, where the cells can be seen with a migratory morphology. (d) NG2 + cells entering the fimbria. (e) Close-up of the inset in $\mathbf{d}$. (f) NG2 + cells surrounding the stem cell graft. The scheme to the right shows the spatial distribution of the images observed in a-f. g, graft; Fi; fimbria; Iv, lateral ventricle; Hi, hippocampus. (g) Immunohistochemical analysis of the migrating NG2 + /Nilo1 + cells. In the image, H2d is in green (Alexa Fluor 488), NG2 in red (Texas Red), Nilo1 is light blue (aminomethyl coumarin acetate) and 4',6-diamidino-2-phenylindole in dark blue. Double-positive NG2 and Nilo1 cells are detected in purple. (h-i) The contralateral side of the fimbria, where no cells were injected, did not present the migrating cells observed in the treated side. Scale bar, $100 \mu \mathrm{m} \mathrm{(c} \mathrm{and} \mathrm{h);} 75 \mu \mathrm{m}(\mathbf{e}) ; 50 \mu \mathrm{m}(\mathbf{a}, \mathbf{b}, \mathbf{d}, \mathbf{f}$ and i); and $25 \mu \mathrm{m}(\mathbf{g})$

Immunofluorescence technique. To fixate the brain tissue, the mice were anesthesized using isoflurane and perfused intracardially $4 \%$ filtered paraformaldehyde (PFA) in phosphate buffer (PB; $\mathrm{pH} 7.4)$. The brain was carefully excised and kept in 4\% PFA overnight. After fixation, the samples were cryoprotected for $12 \mathrm{~h}$ at $4{ }^{\circ} \mathrm{C}$ in $15 \%$ sucrose, followed by incubation overnight in $30 \%$ sucrose. Finally, samples were embedded in Neg-50 Frozen Section Medium (Richard-Allan Scientific, Kalamazoo, MI, USA). Twelve micrometer transverse or longitudinal serial sections were obtained using a Microm HM525 cryostat and mounted on seven parallel series and processed for immunohistochemistry (Microm, Walldorf, Germany). Double-labeled immunohistochemistry was performed using the following primary antibodies: rabbit anti-NG2 (1:150; Santa Cruz Biotechnology, Santa Cruz, CA, USA), mouse anti-MBP (1:100; Oncogene Research Products, La Jolla, CA, USA), rat anti-proteolipid protein (AA3-PLP) (1: 50; Millipore, Billerica, MA, USA), sheep anti-NT3 (1:200; Abcam, Cambridge, UK), sheep anti-NT4/5 (1:200; Chemicon, Millipore, Oxford, UK), sheep anti-NGF$\beta$ (1:500; Chemicon), goat anti-platelet-derived growth factor (PDGF) $(1: 200$; Santa Cruz Biotechnology), hamster anti-Nilo1, ${ }^{20}$ mouse anti-H2D ${ }^{\mathrm{b}}$ haplotype (1:100; BD Pharmingen, San Jose, CA, USA), mouse monoclonal anti-GFP ( $1: 200$, Molecular Probes, Grand Island, NY, USA) and rabbit polyclonal anti-GFP (1:500, Molecular Probes). Even though the grafted stem cells express GFP, immunostaining was performed on this protein in order to amplify the signal, facilitating its observation. As secondary antibodies, for GFP we used anti-mouse or anti-rabbit Alexa Fluor 488 (1:500; Molecular Probes). In the rest of cases, either Alexa Fluor 594 (1:500; Molecular Probes) or biotinylated secondary antibodies were used (1:200; Vector Laboratories, Peterborough, UK) followed by an incubation with streptavidin conjugated with Texas Red or aminomethyl coumarin acetate (1:500; Vector Laboratories). 4',6-Diamidino-2-phenylindole $(2 \mu \mathrm{g} / \mathrm{ml}$; Molecular Probes) was used to stain nuclei. For the expression of NT3, NT4/5, NGF- $\beta$ and PDGF, immunostaining with 3,3'-diaminobenzidine (Applichem, Darmstadt, Germany) was performed. Controls of immunostaining were performed in two slides of each sample following the same procedure, but eliminating primary or secondary antibodies. Histological samples were observed under a fluorescence microscope (Leica DM6000D; Leica Microsystems, Wetzlar, Germany). Micrographs were taken with DFC350/FX and DC500 Leica cameras.

Electrophysiological experiments. The recordings were performed in a preparation of the fimbria-hippocampus 'in vitro' prepared from non-treated, MSCinjected and sham groups, as well as their internal controls. The animals were killed by cervical dislocation at 3 months after grafting or the equivalent age for sham-treated and controls, and the fimbria-hippocampus complex was dissected and placed in a submersion type recording chamber and superfused with extracellular solution (composition in $\mathrm{mM}: \mathrm{NaCl}, 124 ; \mathrm{KCl}, 2.5 ; \mathrm{NaH2PO4,} \mathrm{1.25;}$ 

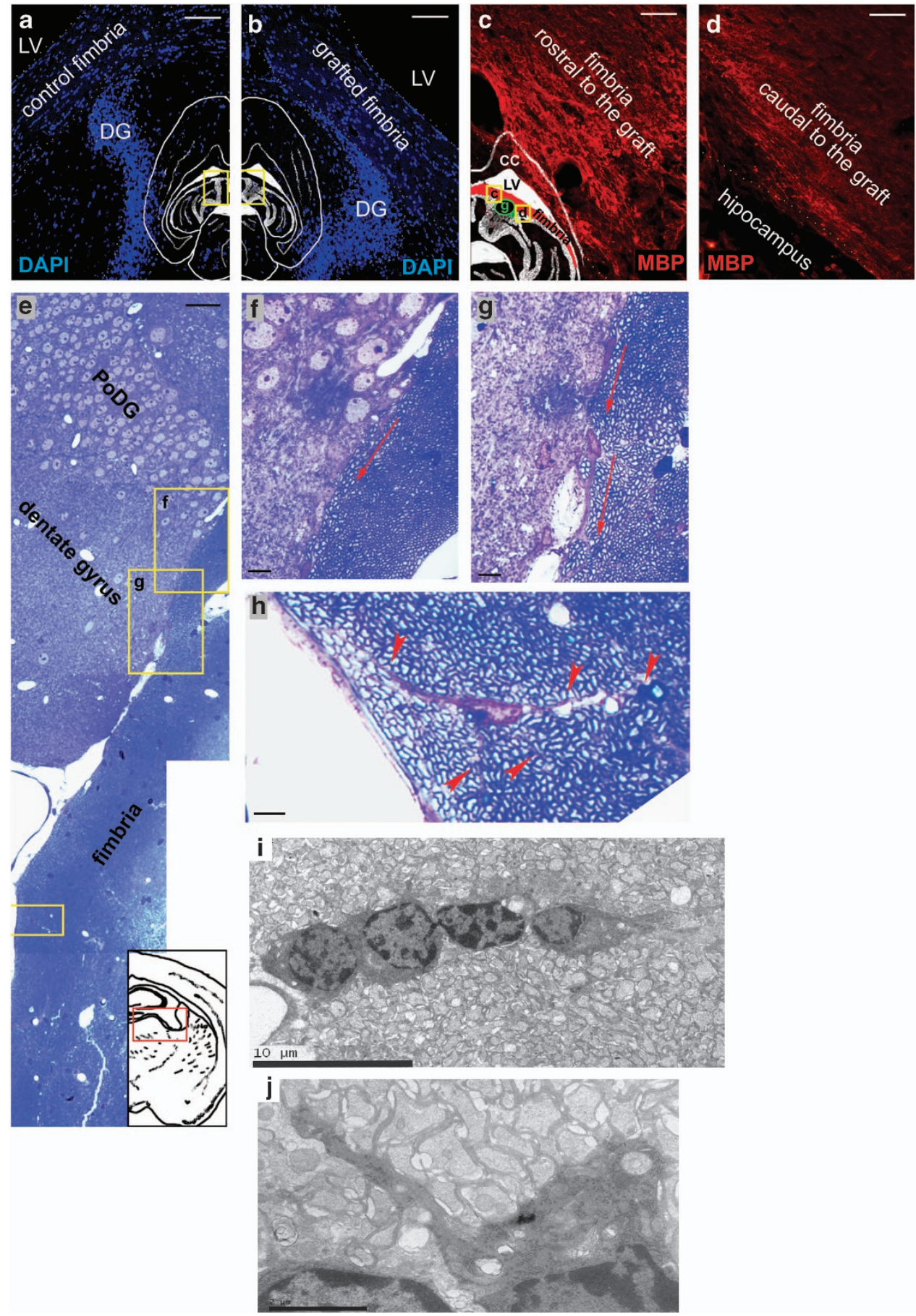

Figure 6 Analysis of neurogenic niches: DG. (a and $\mathbf{b}$ ) Horizontal sections of the contralateral (non-treated) and MSC-grafted side of the fimbria, respectively, showing the tight location of the DG and fimbria, delimited by yellow-dotted lines. Blue is 4',6-diamidino-2-phenylindole staining. (c and d) MBP staining, in red, of the rostral (c) (see ' $r$ ' yellow line in the localization scheme) and caudal (d) regions (see ' $c$ ' yellow line in the localization scheme) to the graft (see ' $g$ ' green line in the localization scheme), showing that the area closest to the DG expresses more MBP. (e) Reconstructed semi-thin ultramicrotome sagittal section showing cells migrating from the DG to the fimbria. (f-h) High magnifications of insets in $\mathbf{e}$. Red arrows in $\mathbf{f}$ and $\mathbf{g}$ indicate cell polarity according to a possible cell migration from the DG. Red arrowheads in $\mathbf{h}$ indicates cytoplasmic prolongations from a oligodendrocyte ensheathing various axons. (i and j) Oligodendrocyte precursors migrating toward the graft, as observed by electron microscopy. Scale bar, $100 \mu \mathrm{m}$ (a and b); $50 \mu \mathrm{m}$ (c-e); $10 \mu \mathrm{m}$ (f and $\mathbf{g}) ;$ and $5 \mu \mathrm{m}$ (h)

NaHCO3, 26; $\mathrm{CaCl} 2,2 ; \mathrm{MgCl} 2,1$; glucose, 10; and osmolarity $300 \mathrm{mOsm} / \mathrm{kg}$ $(\mathrm{pH} 7.4)$, when saturated with $\left.5 \% \mathrm{CO}_{2}+95 \% \mathrm{O}_{2}\right)$. The recordings were obtained

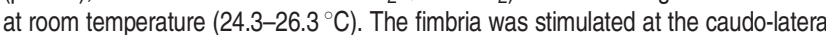
end with a concentric bipolar electrode (Frederick Haer \& Co, Bowdoin, ME, USA) using pulses of $0.1 \mathrm{~ms}$ of duration. Two simultaneous extracellular recordings were obtained with glass pipettes $(0.2-5 \mathrm{M} \Omega$ ) filled with extracellular solution; the two electrodes were placed in parallel with the fimbria axons in different places with respect to the graft. In the grafted fimbria, the graft was identified because of its 


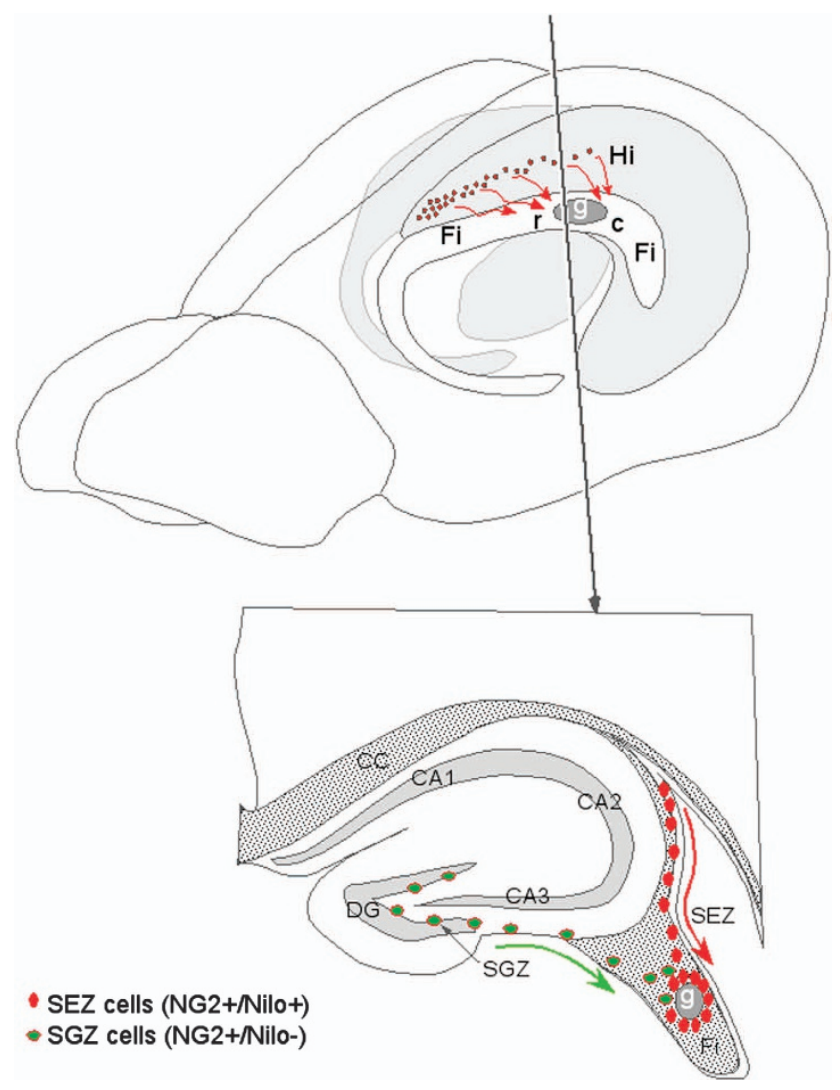

Figure 7 Schematic review of bone marrow-derived MSC-induced remyelination. In the top image, the stem cells (labeled $\mathrm{G}$ ) were grafted into the fimbria (Fi), attracting oligodendrocyte progenitors from the hippocampus $(\mathrm{Hi})$. $\mathrm{R}$ is rostral and $\mathrm{C}$ is caudal. The bottom image depicts a scheme representing the migration of oligodendrocyte progenitors from the two neurogenic niches: the SEZ (red circles) and subgranular zone (SGZ) of the DG (green circles)

native GFP staining, whereas in the sham-operated group the injection site could be detected because of the visible slight scar. The two recording electrodes were placed in four zones located rostro-medial, caudo-lateral, parallel and inside, with respect to the graft (in MSC group); in the sham group or internal control group, we used equivalent recording positions. The conduction velocity was measured from the distance between the tips of both electrodes and the difference in the latency of the propagated action potential.

Transmission electron microscopy (TEM) studies. For TEM, the mice were perfused with $2 \%$ PFA $/ 1.5 \%$ glutaraldehyde in $\mathrm{PB} 0.12 \mathrm{M}$ with $\mathrm{Cl}_{2} \mathrm{Ca}$. Brains were post-fixed in the same fixative overnight at $4{ }^{\circ} \mathrm{C}$, and fimbria was dissected and washed for $12 \mathrm{~h}$ in PB $0.1 \mathrm{M}(\mathrm{pH} 7.4)$ with $7 \%$ sucrose. Afterwards, the fimbria were post-fixed in $1 \%$ osmium tetraoxide in $0.2 \mathrm{M}$ cacodilate buffer, rinsed and stained with $2.5 \%$ uranile acetate in Michaelis buffer. Samples were dehydrated with acetone and propylene oxide and embedded in EPON. Thick sections $(1 \mu \mathrm{m})$ were obtained rostro-medially, caudo-laterally and at the level of the graft. Ultra-thin electron miscroscope images were observed by the EM department in the University of Murcia, Spain.

Quantification of immunoreactivity in the fimbria. The quantification of GFAP, MBP and NG2 immunoreactivity in the fimbria was performed using imaging analysis software (ImageJ). To do so, one image was taken of the whole fimbria per serial section (one out of every seven consecutive sections) and changed to an 8-bit image (becoming a black and white image). Then, the fimbria was delimited and the percentage of illuminated pixels (white pixels) was calculated, following the instructions published in Hartig. ${ }^{42}$ Finally, the average and S.D. of the seven percentages were calculated. This was performed both in the sham-operated and stem cell-treated groups and compared. In the case of the expression of the trophic factors analyzed in the fimbria, these were quantified in a similar fashion, only that they were normalized to the contralateral (non-treated) side of the fimbria. In this manner, a value over 1 indicated that the treated side presented higher immunoreactivity compared with the non-treated side.

PCR analysis. Cultured MSCs were analyzed for gene expression of neurotrophic factors NT3, NT4/5, PDGF and NGF- $\beta$. Total RNA was extracted using the RNeasy mini kit (Qiagen, Venlo, Netherlands) and DNase I treatment to avoid genomic contamination. Reverse Transcription was performed using the Quantitect Reverse Transcription kit (Qiagen) and processed using the Qiagen Multiplex PCR kit. The sequences of the primers used were: NT3 forward 5'-AGTTTGCCGGAAGACTCTCTC-3', reverse $5^{\prime}$-GGGTGCTCTGGTAATTT TCCTTA-3'; NT4/5 forward 5'-TGAGCTGGCATATGCGAC, reverse 5'-CAGCGC GTCTCGAAGAAGT-3'; PDGF forward 5'-GCAAGACCAGGACGGTCATTT, reverse $5^{\prime}$-GGCACTTGACACTGCTCGT- $3^{\prime}$; and NGF- $\beta$ forward $5^{\prime}$-GCACTA CACCCATCAAGTTCA- $3^{\prime}$, reverse $5^{\prime}$-TCCTGAGTCATGCTCACAAGT-3'. The resulting PCR products were placed in a QIAxcel apparatus (Qiagen) and analyzed using the QIAxcel gel analysis software (QIAxcel Biocalculator).

Statistical analysis. Student's t-test for independent samples was used to detect significant differences between sham and MSC-grafted or MSC-grafted and control fimbria for each case, using IBM SPSS Statistic v19.0.0 software (Armonk, NY, USA).

\section{Conflict of Interest}

The authors declare no conflict of interest.

Acknowledgements. This work was supported by MINISTERIO CIENCIA E INNOVACION (MICINN BFU2010-27326), DIGESIC-MEC BFU2008-00588, Ingenio 2010 MEC-CONSOLIDER CSD2007-00023, from the GVA Prometeo grant 2009/028 and ISCIII Cibersam and Tercel (RD06/0010/0023), as well as ELA Foundation, Fundación Diogenes-Elche city government and Rotary Club Elche-lllice and Fundació Gent per Gent grant 24 NEURO.

1. Steinman L. Multiple sclerosis: a coordinated immunological attack against myelin in the central nervous system. Cell 1996; 85: 299-302.

2. Aktas $\mathrm{O}$, Kieseier $\mathrm{B}$, Hartung HP. Neuroprotection, regeneration and immunomodulation: broadening the therapeutic repertoire in multiple sclerosis. Trends Neurosci 2010; 33 140-152.

3. Kohler W. Leukodystrophies with late disease onset: an update. Curr Opin Neurol 2010; 23: 234-241.

4. Nave KA. Myelination and support of axonal integrity by glia. Nature 2010; 468: 244-252.

5. Nishiyama A, Komitova M, Suzuki R, Zhu X. Polydendrocytes (NG2 cells): multifunctional cells with lineage plasticity. Nat Rev Neurosci 2009; 10: 9-22.

6. Sellers DL, Maris DO, Horner PJ. Postinjury niches induce temporal shifts in progenitor fates to direct lesion repair after spinal cord injury. J Neurosci 2009; 29: 6722-6733.

7. Menn B, Garcia-Verdugo JM, Yaschine C. Origin of oligodendrocytes in the subventricular zone of the adult brain. J Neurosci 2006; 26: 7907-7918.

8. Chari DM, Crang AJ, Blakemore WF. Decline in rate of colonization of oligodendrocyte progenitor cell (OPC)-depleted tissue by adult OPCs with age. J Neuropathol Exp Neurol 2003; 62: 908-916.

9. Chari DM, Huang WL, Blakemore WF. Dysfunctional oligodendrocyte progenitor cell (OPC) populations may inhibit repopulation of OPC depleted tissue. J Neurosci Res 2003; 73: 787-793.

10. Chari DM. Remyelination in multiple sclerosis. Int Rev Neurobiol. 2007; 79: 589-620.

11. Williams A, Piaton G, Aigrot MS, Belhadi A, Théaudin M, Petermann F et al. Semaphorin 3A and 3F: key players in myelin repair in multiple sclerosis? Brain 2007; 130: 2554-2565

12. Kuhlmann T, Miron V, Cui $Q$ Wegner $C$, Antel J, Bruck W. Differentiation block of oligodendroglial progenitor cells as a cause for remyelination failure in chronic multiple sclerosis. Brain 2008; 131: 1749-1758.

13. Rivera FJ, Steffenhagen C, Kremer D, Kandasamy M, Sandner B, Couillard-Despres $S$ et al. Deciphering the oligodendrogenic program of neura progenitors: cell intrinsic and extrinsic regulators. Stem Cells Dev 2010; 19: 595-606.

14. Zhang J, Li Y, Chen J, Cui Y, Lu M, Elias SB et al. Human bone marrow stromal cell treatment improves neurological functional recovery in EAE mice. Exp Neurol 2005; 195: 16-26.

15. Zhang J, LiY, Lu M, Cui Y, Chen J, Noffsinger L et al. Bone marrow stromal cells reduce axona loss in experimental autoimmune encephalomyelitis mice. J Neurosci Res 2006; 84: 587-595.

16. Cristofanilli M, Harris VK, Zigelbaum A, Goossens AM, Lu A, Rosenthal $\mathrm{H}$ et al. Mesenchymal stem cells enhance the engraftment and myelinating ability of allogeneic oligodendrocyte progenitors in dysmyelinated mice. Stem Cells Dev 2011; 20: 2065-2076. 
17. Rivera FJ, Kandasamy M, Couillard-Despres S, Caioni M, Sanchez R, Huber C et al. Oligodendrogenesis of adult neural progenitors: differential effects of ciliary neurotrophic factor and mesenchymal stem cell derived factors. J Neurochem 2008; 107: 832-843.

18. Steffenhagen C, Dechant FX, Oberbauer E, Furtner T, Weidner N, Küry $P$ et al. Mesenchymal stem cells prime proliferating adult neural progenitors toward an oligodendrocyte fate. Stem Cell Dev 2012; 21: 1838-1851.

19. Kumar S, Biancotti JC, Yamaguchi M, de Vellis J. Combination of growth factors enhances remyelination in a cuprizone-induced demyelination mouse model. Neurochem Res 2007; 32: 783-797.

20. Del Valle I, Elvira G, Garcia-Benzaquen L. Characterization of novel monoclonal antibodies able to identify neurogenic niches and arrest neurosphere proliferation and differentiation. Neuroscience 2010; 169: 1473-1485.

21. Sim FJ, Zhao C, Penderis J, Franklin RJ. The age-related decrease in CNS remyelination efficiency is attributable to an impairment of both oligodendrocyte progenitor recruitment and differentiation. J Neurosci 2002; 22: 2451-2459.

22. Armstrong RC, Le TQ, Flint NC, Vana AC, Zhou YX. Endogenous cell repair of chronic demyelination. J Neuropathol Exp Neurol 2006; 65: 245-256.

23. Nishiyama A, Lin XH, Giese N, Heldin $\mathrm{CH}$, Stallcup WB. Interaction between NG2 proteoglycan and PDGF alpha-receptor on $\mathrm{O} 2 \mathrm{~A}$ progenitor cells is required for optimal response to PDGF. J Neurosci Res 1996; 43: 315-330.

24. McKinnon RD, Matsui T, Aranda M, Dubois-Dalcq M. A role for fibroblast growth factor in oligodendrocyte development. Ann NY Acad Sci 1991; 638: 378-386.

25. Barres BA, Raff MC, Gaese F, Bartke I, Dechant G, Barde YA. A crucial role for neurotrophin-3 in oligodendrocyte development. Nature 1994; 367: 371-375

26. Ye P, D'Ercole AJ. Insulin-like growth factor I protects oligodendrocytes from tumor necrosis factor-alpha-induced injury. Endocrinology 1999; 140: 3063-3072.

27. Barres BA, Schmid R, Sendnter M, Raff MC. Multiple extracellular signals are required for long-term oligodendrocyte survival. Development 1993; 118: 283-295.

28. Jiang F, Frederick TJ, Wood TL. IGF-I synergizes with FGF-2 to stimulate oligodendrocyte progenitor entry into the cell cycle. Dev Biol 2001; 232: 414-423.

29. Wolswijk G, Noble M. Cooperation between PDGF and FGF converts slowly dividing O-2A adult progenitor cells to rapidly dividing cells with characteristics of $\mathrm{O}-2 \mathrm{~A}$ perinatal progenitor cells. J Cell Biol 1992; 118: 889-900.

30. Boyd JG, Gordon T. A dose-dependent facilitation and inhibition of peripheral nerve regeneration by brain-derived neurotrophic factor. Eur J Neurosci 2002; 15: 613-626.

31. Beck M, Flachenecker P, Magnus T, Giess R, Reiners K, Toyka KV et al. Autonomic dysfunction in ALS: a preliminary study on the effects of intrathecal BDNF. Amyotroph Lateral Scler Other Motor Neuron Disord 2005; 6: 100-103.
32. Eriksdotter-Jönhagen $M$, Nordberg $A$, Amberla $K$, Bäckman $L$, Ebendal $T$, Meyerson $B$ et al. Intracerebroventricular infusion of nerve growth factor in three patients with Alzheimer's disease. Dement Geriatr Cogn Disord 1998; 9: 246-257.

33. Nutt JG, Burchiel KJ, Comella CL, Jankovic J, Lang AE, Laws ER Jr. et al. Randomized, double-blind trial of glial cell line-derived neurotrophic factor (GDNF) in PD. Neurology 2003; 60: 69-73

34. Caplan Al, Dennis JE. Mesenchymal stem cells as trophic mediators. J Cell Biochem 2006; 98: 1076-1084

35. Uccelli A, Moretta L, Pistoia V. Immunoregulatory function of mesenchymal stem cells. Eur J Immunol 2006; 36: 2566-2573.

36. Rivera FJ, Siebzehnrubl FA, Kandasamy M, Couillard-Despres S, Caioni M, Poehler AM et al. Mesenchymal stem cells promote oligodendroglial differentiation in hippocampal slice cultures. Cell Physiol Biochem 2009; 24: 317-324.

37. Levine JM, Reynolds R, Fawcett JW. The oligodendrocyte precursor cell in health and disease. Trends Neurosci 2001; 24: 39-47.

38. Franklin RJ. Why does remyelination fail in multiple sclerosis? Nat Rev Neurosci 2002; 3: 705-714.

39. Nishiyama A, Yang Z, Butt A. Astrocytes and NG2-glia: what's in a name? J Anat 2005; 207: 687-693.

40. Jones J, Jaramillo-Merchan J, Bueno C, Pastor D, Viso-Leon MC, Martinez S. Mesenchymal stem cells rescue Purkinje cells and improve motor functions in a mouse model of cerebellar ataxia. Neurobiol Dis 2010; 40: 415-423.

41. Pastor D, Viso-Leon MC, Jones J, Jaramillo-Merchán J, Toledo-Aral JJ, Moraleda JM et al. Comparative effects between bone marrow and mesenchymal stem cell transplantation in GDNF Expression and motor function recovery in a motorneuron degenerative mouse model. Stem Cell Rev Rep 2012; 8: 445-458.

42. Hartig SM. Basic image analysis and manipulation in ImageJ. Curr Protoc Mol Biol 2013 Chapter 14: Unit 14.15; 1-14

(c) (i) () $\Theta$ Cell Death and Disease is an open-access journal published by Nature Publishing Group. This work is licensed under a Creative Commons Attribution-NonCommercialNoDerivs 3.0 Unported License. To view a copy of this license, visit http://creativecommons.org/licenses/by-nc-nd/3.0/ 